This is the peer reviewed accepted manuscript of the following article:

Poli A, Fiume R, Baldanzi G, Capello D, Ratti S, Gesi M, Manzoli L, Graziani A, Suh PG, Cocco L, Follo MY.

Nuclear Localization of Diacylglycerol Kinase Alpha in K562 Cells Is Involved in Cell Cycle Progression.

J Cell Physiol. 2017 Sep;232(9):2550-2557

Final peer reviewed version available at: https://doi.org/10.1002/jcp.25642

Rights / License:

The terms and conditions for the reuse of this version of the manuscript are specified in the publishing policy. For all terms of use and more information see the publisher's website.

This item was downloaded from IRIS Università di Bologna (https://cris.unibo.it/)

When citing, please refer to the published version. 


\section{Nuclear localization of Diacylglycerol Kinase alpha in K562 cells is involved in cell cycle progression $^{\dagger}$}

Alessandro Poli ${ }^{1,2,3}$, Roberta Fiume ${ }^{1}$, Gianluca Baldanzi ${ }^{4}$, Daniela Capello ${ }^{4}$, Stefano Ratti ${ }^{1}$, Marco Gesi $^{5}$, Lucia Manzoli ${ }^{1}$, Andrea Graziani ${ }^{4,6}$, Pann-Ghill Suh ${ }^{7}$, Lucio Cocco ${ }^{1,{ }^{*}}$, Matilde Y. Follo ${ }^{1,{ }^{*}}$

${ }^{1}$ Cellular Signalling Laboratory, Department of Biomedical and Neuromotor Sciences, University of Bologna; "Istituto Nazionale Genetica Molecolare "Romeo e Enrica Invernizzi", Milano. Italy; ${ }^{3}$ Department of Medical Biotechnology and Translational Medicine, Università degli Studi di Milano, Milan, Italy; ${ }^{4}$ Department of Translational Medicine and Institute for Research and Cure of Autoimmune Diseases, University of Piemonte Orientale, Novara, Italy; ${ }^{5}$ Department of Translational Research and New Technologies in Medicine and Surgery, University of Pisa, Italy; ${ }^{6}$ University Vita e Salute San Raffaele, Milan, Italy; ${ }^{7}$ School of Life Sciences, Ulsan National Institute of Science and Technology, Ulsan, Republic of Korea

*Corresponding Authors: Dr. Lucio Cocco and Dr. Matilde Y. Follo, Cellular Signalling Laboratory, Institute of Human Anatomy, Department of Biomedical and Neuromotor Sciences, University of Bologna, via Irnerio 48, 40126 Bologna, Italy. Phone: +39-0512091511; Fax: +39-051251735; email: lucio.cocco@unibo.it; matilde.follo@unibo.it

Running Head: Nuclear DGKalpha and cell cycle in K562 cells

Keywords: Phosphatidylinositol; Diacylglycerol Kinase; Cell Cycle; K562 Cells; Nucleus

Contract grant sponsor: Intesa San Paolo (to LC); AIRC IG 13524 (to AG) 


\begin{abstract}
Phosphatidylinositol (PI) signalling is an essential regulator of cell motility and proliferation. A portion of PI metabolism and signalling takes place in the nuclear compartment of eukaryotic cells, where an array of kinases and phosphatases localize and modulate PI. Among these, Diacylglycerol Kinases (DGKs) are a class of phosphotransferases that phosphorylate diacylglycerol and induce the synthesis of phosphatidic acid. Nuclear DGKalpha modulates cell cycle progression, and its activity or expression can lead to changes in the phosphorylated status of the Retinoblastoma protein, thus impairing G1/S transition and, subsequently, inducing cell cycle arrest, which is often uncoupled with apoptosis or autophagy induction. Here we report for the first time not only that the DGKalpha isoform is highly expressed in the nuclei of human erythroleukemia cell line K562, but also that its nuclear activity drives $\mathrm{K} 562$ cells through the G1/S transition during cell cycle progression.
\end{abstract}




\section{Introduction}

Phosphatidylinositol (PI) is a lipid molecule characterized by a hydrophilic head group (inositol ring) bound to a hydrophobic tail (glycerol and two fatty acid chains) through a phosphodiester link. PI is involved in many signalling pathways and its metabolism regulates important cellular processes including cell cycle, cell differentiation, membrane trafficking and gene expression (Cocco et al., 2015b). An array of kinases and phosphatases capable to modulate PI, adding or removing phosphate groups on the inositol ring, composes the so called PI cycle, and evidences of PIs and enzymes localized in the nuclear compartment have been described (Blind, 2014). This commonly known nuclear PI metabolism usually includes Phospholipase C (PLC), a class of enzymes able to hydrolyze Phosphatidylinositol 4,5-biphosphate (PI(4,5)P2) to Inositol 1,4,5trisphosphate $(\operatorname{Ins}(1,4,5) P 3)$ and Diacylglycerol (DAG), important second messengers involved in the activation of Protein Kinases C (PKCs) and phosphorylated by Diacylglycerol Kinases (DGKs) (Baldanzi, 2014; Follo et al., 2015; Poli et al., 2014a; Tanaka et al., 2016; Tu-Sekine et al., 2015).

DGKs are a family of ten proteins divided into five classes according to their specific domain composition. All the isoforms share the same catalytic activity: the synthesis of phosphatidic acid (PA) through DAG phosphorylation, using ATP as the source of the phosphate (Baldanzi, 2014). This event regulates different cellular functions, such as cell cycle progression and cell differentiation (Almena et al., 2013; Goto et al., 2014; Merida et al., 2008). As a complete PI cycle resides in the nucleus of eukaryotic cells, evidences collected by many studies indicated that the DGK activity can also be found in the nuclear matrix, suggesting the involvement of DGKs in nuclear processes, and particularly in cell cycle progression (Divecha et al., 1993; Payrastre et al., 1992; Raben and Tu-Sekine, 2008; Shulga et al., 2011). For instance, DGKzeta is a negative regulator of cell cycle progression, as its overexpression inhibits the entry into $\mathrm{S}$ phase of cell cycle, while the silencing or inhibition its enzymatic activity accelerates cell cycle (Evangelisti et al., 2007; Topham et al., 1998). In addition, DGKzeta regulates G1/S transition through its interaction with the Retinoblastoma protein (pRb) (Los et al., 2006). On the other hand, overexpression of a kinasedead mutant of nuclear DGKgamma negatively affects $S$ phase progression, and also nuclear DGKalpha can play a putative role in cell cycle regulation (Matsubara et al., 2006).

DGKalpha can shuttle inside and outside the nucleus of different cell types under specific stimuli (Matsubara et al., 2012; Wada et al., 1996), and specific factors may either stimulate or inhibit its enzymatic activity. For instance, both in interleukin-2 (IL-2)-stimulated lymphocytes and in lymphoma cells expressing the NPM-ALK oncogene, activation of DGKalpha positively affected cell 
cycle entry and proliferation (Bacchiocchi et al., 2005; Flores et al., 1996; Flores et al., 1999). Conversely, in T cells, the inhibition of DGKalpha induced by the T-cell receptor (TCR) amplified the TCR-induced response by enhancing the DAG-mediated signalling (Baldanzi et al., 2011; Ruffo et al., 2016). This different regulation and biological role of DGKalpha may be due to a specific subcellular distribution of DGKalpha that regulates different PA-dependent pathways, as demonstrated in $\mathrm{K} 562$ cells, where the nuclear localization of DGKalpha specifically regulates cell cycle (Poli et al., 2013; Poli et al., 2014b).

Here, we report that the modulation of nuclear DGKalpha in K562 cells, through gene manipulation and specific DGK inhibitors, is indeed involved in cell proliferation by control of $p R b$ phosphorylation and in cell cycle progression through the $\mathrm{G} 1 / \mathrm{S}$ checkpoint.

\section{Materials and Methods}

\section{Antibodies and reagents}

The following antibodies and reagents were purchased from commercial sources. DGKalpha (Abcam, \#64845); Cyclin B1 (Santa Cruz Biotechnology; \#sc-245); p-pRb 780 (Sigma-Aldrich; \#R6275) and Beta-tubulin (Sigma-Aldrich; \#T7816); pRb (Cell Signalling Technology; \#9309), p-pRb 795 (Cell Signalling Technology; \#9301s), p-pRb 807/811 (Cell Signalling Technology; \#9308), Cyclin E (Cell Signalling Technology; \#4129s), Cyclin D3 (Cell Signalling Technology; \#2936), Caspase-9 (Cell Signalling Technology; \#9502), Caspase-3 (Cell Signalling Technology; \#9662), Beclin-1 (Cell Signalling Technology; \#3495), LC-3b (Cell Signalling Technology; \#2775) and Histone 2A (H2A, Cell Signalling Technology; \# 2718). Secondary antibodies were horseradish peroxidase (HRP)conjugated anti-rabbit IgG (Cell Signalling Technology; \#7074,) and HRP-conjugated anti-mouse IgG (Cell Signalling Technology; \#7076). The Phototope ${ }^{\circledR}$-HRP Western Blot Detection System (Cell Signalling Technology; \#7071) was also used.

\section{Tissue cell cultures}


Human erythroleukemia K562 cells were grown in RPMI 1640 (Sigma-Aldrich) supplemented with $10 \%$ heat-inactivated fetal bovine serum (FBS), L-glutamine and penicillin/streptomycin. Cells were treated with the following compounds: DGK inhibitor I (Sigma-Aldrich, \#R59022) and DGK inhibitor II (Sigma-Aldrich, \#R59949) for 24, 48 and 72 hours.

\section{MTT assay}

Cells were seeded at a final density of $2 \times 10^{4}$ cells/well in a 96-well plate. DGK inhibitor I and II were added for $48 \mathrm{~h}$ at increasing concentrations. Proliferating cells cultivated in complete RPMI 1640 with $10 \%$ FBS were used as the control, whereas wells filled with only the medium were considered as the background. MTT experiments were performed following the manufacturer's instructions (Life Technologies). Data were analyzed reading the absorbance at $570 \mathrm{~nm}$. All results were compared with the control samples after subtraction of the values given by the background. Results were statistically analyzed by GraphPad Prism software (v. 6.0) and Student's t-test was used to compare continuous values.

\section{Cell Counting}

To determine the rate of cell growth, cells were seeded at a cell density of $2.5 \times 10^{4}$ cells $/ \mathrm{ml}$ in 12 well plates and, then, treated with DGK inhibitor I and DGK inhibitor II (Sigma-Aldrich). In the experiments of gene manipulation, cells were transfected to overexpress/silence DGKalpha, or with an empty vector/scrambled siRNA as control, and 2 hours later seeded in complete RPMI $164010 \%$ FBS at the same cell density $\left(2.5 \times 10^{4}\right.$ cells $/ \mathrm{ml}$ in 12 -well plates $)$. Cell growth was determined by direct counting of cells harvested at 24,48 and 72 hours after seeding. Viable cells were counted by a hemocytometer using $0.2 \%$ Trypan Blue. Results were statistically analyzed by GraphPad Prism software (v. 6.0) and Student's t-test was used to compare continuous values.

\section{Cell cycle synchronization}


Growing cells were collected and synchronized: (1) G1/S cells: proliferating cells were cultured in complete RPMI 1640 with FBS 10\%; (2) G2/M blocked cells: growing cells were treated with Nocodazole (Sigma-Aldrich) at a final concentration of $20 \mathrm{ng} / \mathrm{ml}$ for 16 hours; (3) G0/G1 cells: growing cells were synchronized at G2/M by $20 \mathrm{ng} / \mathrm{ml}$ Nocodazole treatment for 16 hours before being released for 3 hours in complete RPMI 1640 10\% FBS.

\section{Cell transfection}

Cells were seeded at a cell density of $5 \times 10^{5}$ cells $/ \mathrm{ml}$ in 6-well plates, then cells were transfected with full-length DNA vectors for human DGKalpha cloned into YFP-pDEST 6.2, using an empty pcDNA/2.1 plasmid (Invitrogen) as a control. Conversely, the expression of DGKalpha was silenced by a specific siRNA (Applied Biosystems) at a final concentration of $50 \mathrm{nM}$, using the electroporation assay kit (Tema Ricerca, program T-16) as indicated by the manufacturers' instructions, while a mix of Silencer Select Negative Control \#1 and \#2 siRNAs (Applied Biosystems) was used as a negative control. 24 hours after transfection (overexpression/silencing), K562 cells were treated with both DGK inhibitor I and DGK inhibitor II at a final concentration of $20 \mu \mathrm{M}$ for 24 hours.

\section{Flow cytometric analysis of cell cycle}

For cell cycle analysis, cells were cultivated in RPMI medium with the addition of DGK inhibitor I and DGK inhibitor II (Sigma-Aldrich), and the analysis was performed as reported elsewhere (Chiarini et al., 2010). At least 10,000 events/sample were acquired. Results were statistically analyzed by GraphPad Prism software (v. 6.0) and Student's t-test was used to compare continuous values.

\section{Annexin $\mathrm{V} / \mathrm{PI}$ assay}

Annexin V/PI analyses were performed as indicated by the manufacturer's instructions (Life Technologies), as previously reported (Lonetti et al., 2014). Cells were treated with DGK inhibitor I and DGK inhibitor II at a final concentration of $20 \mu \mathrm{M}$ for 48 hours. Then, the assay was performed 
acquiring at least 5000 events per sample. Results were statistically analyzed by GraphPad Prism software (v. 6.0) and Student's t-test was used to compare continuous values.

\section{Analysis of DGKalpha Gene Expression}

Total RNA was extracted and retro-transcribed as previously reported (Cocco et al., 2015a). The expression of DGKalpha gene was assessed using a standard TaqMan Real-Time PCR method (Applied Biosystems), with the 18s TaqMan probe as the endogenous control (Applied Biosystems). Results were statistically analyzed by GraphPad Prism software (v. 6.0) and Student's t-test was used to compare continuous values.

\section{Analysis of Protein Expression}

Protein amount was assessed by Western Blot analyses, and nuclear and cytoplasmic fractionation was performed as already reported (Blalock et al., 2011). Beta-tubulin was used as a control of equal protein loading. Purity of nuclear and cytoplasmic fractionations were controlled using Betatubulin (cytoplasmic marker) and H2A (nuclear marker) antibodies.

\section{Results}

\section{Nuclear localization of DGKalpha isoform in K562 cells}

As Figure 1a shows, K562 cells were induced to a specific and complete synchronization at the three different checkpoints. The levels of DGKalpha were then investigated in both cytoplasmic and nuclear fractions of these synchronized cells. As Figure $1 \mathrm{~b}$ shows, DGKalpha appeared to be mainly localized inside the nucleus of $\mathrm{K} 562$ cells and, even if slightly, showed a peak at the G2/M checkpoint. On the contrary, DGKalpha was barely expressed in the cytoplasmic fraction of this cell line (Figure 1b). 
Both DGK inhibitor I and II, also called R59022 and R59949 respectively, inhibit DGKalpha activity with a IC50 of about $20 \mu \mathrm{M}$ (Sato et al., 2013). In a dose-response MTT assay, both DGK inhibitors significantly downregulated cell proliferation at concentrations higher than $10 \mu \mathrm{M}$ and an $\mathrm{ED}_{50}$ of $20 \mu \mathrm{M}$, whereas higher concentrations drove to a very strong decrease of the MTT signal, suggesting a toxic effect (Figure 2a). Cell growth analyses completely matched the MTT data, showing no changes in cell proliferation by treatment with DGK inhibitor I or II at final concentrations up to $10 \mu \mathrm{M}$, whereas doses equal or higher than $20 \mu \mathrm{M}$ appeared to slow down proliferation (Figure $2 b$ ).

K562 cells treated with DGK inhibitors I and II show accumulation in the G0/G1 phase of cell cycle and a reduced phosphorylation of $\mathrm{pRb}$

When compared with proliferating cells, the treatment with both DGK inhibitors induced a relevant accumulation of $\mathrm{K} 562$ cells at the G0/G1 phase of cell cycle and a consistent reduction of cells in both $\mathrm{S}$ and $\mathrm{G} 2 / \mathrm{M}$ phases (Figure $2 \mathrm{c}$ ).

Moreover, also the expression of some proteins regulating cell cycle machinery was screened by Western blot (Figure 2d). Interestingly, DGK inhibitor I and DGK inhibitor II led to important decrease of the phosphorylation status of $\mathrm{pRb}$, while the levels of the total protein did not change. On the other hand, Cyclin E seemed not affected, whereas a slight increase of Cyclin D3 and a strong downregulation of Cyclin B1 were found (Figure 2d).

Impaired cell cycle by inhibition of DGK signalling is not linked to upregulation of apoptosis or autophagy

In order to further investigate the role of DGKalpha in cell cycle regulation, we treated K562 cells with the two DGK inhibitors for 48 hours and, then, we collected and stained the cells with Annexin V/PI in order to quantify the apoptotic (Annexin $\mathrm{V}+$ ) or necrotic $(\mathrm{PI}+$ ) ones. Surprisingly, 48 hours of treatment with $20 \mu \mathrm{M}$ of either DGK inhibitor I or II led to cell accumulation at G0/G1, without affecting the percentage of apoptotic or necrotic cells (Figure 3a), and the expression of both full-length and cleaved Caspases 9 and 3 (Figure 3b). Finally, no changes in the levels of Beclin-1 and LC3b, essential regulators of authophagy, were observed (Figure 4). 


\section{Modulation of nuclear DGKalpha affects cell cycle progression through the G1/S transition}

In order to evaluate the specific role of DGKalpha isoform, we knocked down its expression by transfecting K562 with a specific DGKalpha siRNA. As shown in Figure 5a, qPCR and Western blot analyses confirmed the downregulation of DGKalpha mRNA and protein in K562 cells. In particular, we found that upon DGKalpha knock down, cell proliferation was significantly reduced if compared with the scrambled controls (Figure 5b). Next, cell cycle profiling was investigated. Strikingly, $48 \mathrm{~h}$ after the transfection, DGKalpha knocked down cells showed an important accumulation at the G0/G1 phase (Figure $5 c$ ).

On the other hand, the overexpression of DGKalpha (DGKalpha OV) led to a relevant increase of the protein levels in K562 cells (Figure $5 \mathrm{~d}$ ) and cell proliferation assays showed a slight increase in overexpressing cells (Figure 5e). In addition, 48 hours after the transfection, cell cycle analyses indicated a significant increase of cells overexpressing DGKalpha in the S phase, as compared with control cells (Figure 5f). Subsequently, we overexpressed DGKalpha and, 24 hours later, we treated K562 cells with DGK inhibitor I or DGK inhibitor II for further 24 hours. Cell cycle analyses indicated that the effects of inhibition of cell cycle progression by both DGK inhibitor I and II were partially limited by overexpression of DGKalpha. Indeed, as shown in Figure 6, DGKalpha upregulation led the cells to partially overcome the G0/G1 block, leading to a significant increase in the percentage of cells in $\mathrm{S}$ phase.

\section{Discussion}

Many reports indicate that DGKs are localized also in the nucleus, where they may phosphorylate nuclear DAG to generate PA, making these enzymes as part of nuclear PI metabolism (Payrastre et al., 1992; Raben and Tu-Sekine, 2008; Vann et al., 1997). On the other hand, oscillations of the nuclear pool of PIs during cell proliferation, including PA synthesis, have been observed as able to modulate cell cycle progression (Bulley et al., 2015; Giudici et al., 2016; Irvine, 2003; Shulga et al., 2011). In this study we focused on a specific DGK isoform, DGKalpha, whose localization in the nucleus was detectable in specific cell types (Raben and Tu-Sekine, 2008). Despite some preliminary evidences suggested that nuclear DGKalpha is involved in nuclear PI metabolism and 
in cell proliferation, its role still remains to be fully elucidated (Bacchiocchi et al., 2005; Baldanzi et al., 2011; Flores et al., 1996; Flores et al., 1999). We recently described that a robust nuclear DAG increase, due to nuclear PLCbeta1 activity, drives human K562 erythroleukemia cell line through the G2/M checkpoint of cell cycle (Poli et al., 2014b). Moreover, D'Santos et al. showed that, in K561 cells, the down- and up-regulation of nuclear DAG and PA contribute to overpass mitosis and induce cells to enter the G0/G1 phase, thus suggesting a key role for DGK enzymes in this process (D'Santos et al., 1999).

Stemming from these data, here we intended to elucidate the contribution of DGKalpha, which is highly expressed in the nucleus of $\mathrm{K} 562$ cells, in nuclear PI modulation, by carrying out DAG phosphorylation to PA, and we were also interested in investigating the putative role of DGKalpha in driving cells through the G0/G1 checkpoint and enter into the $S$ phase during cell cycle progression and cell proliferation.

Our data support the notion that DGKalpha is part of a well-defined nuclear PI metabolism able to affect cell cycle and cell proliferation of K562 human erythroleukemia cell line. Indeed, the participation of DGK activity in nuclear PI metabolism is known and several DGK isoforms, such as DGKtheta and DGKzeta, have been observed in the nucleus (Evangelisti et al., 2007; Merida et al., 2008; Shulga et al., 2011). While DGKalpha is known to shuttle between the nucleus and cytoplasm/plasma membrane (Baldanzi et al., 2011; Matsubara et al., 2012), here we report for the first time that this specific isoform is strongly localized into the nucleus of $\mathrm{K} 562$ erythroleukemia cells. Indeed, synchronization experiments at different cell cycle checkpoints showed an important expression of DGKalpha in the nuclear fraction of this cell model, slightly peaking at G2/M. This suggested that DGKalpha might have a function in nuclear signalling. In particular, we observed that the inhibition of DGK signalling by treatment with DGK inhibitor I and DGK inhibitor II can down-modulate $\mathrm{K} 562$ cell proliferation, specifically affecting G1/S transition. Cells treated with both these compounds were indeed characterized by an important block at the G0/G1 checkpoint and a parallel reduction of the $\mathrm{S}$ and G2/M phases. This impaired cell cycle phenotype was due to a strong decrease of the levels of phosphorylation of $p R B$, which is an essential process for the detachment of pRb from E2F and, in turn, for cell cycle entry into $S$ phase. Moreover, we also observed a slight increase of Cyclin D3 and an important reduction of Cyclin B1 expression, linked with the peculiar $\mathrm{G} 1$ accumulation and the diminished G2/M phase in the cells. Interestingly, the G0/G1 block obtained by DGK inhibition did not lead to induction of apoptosis or autophagy. 
Furthermore, as we have previously documented the relevance of nuclear PI cycle for cell proliferation in K562 cells (Poli et al., 2013; Poli et al., 2014b), given that DGKalpha has a strong nuclear localization in this cell model, and considering that both DGK inhibitor I and II can specifically inhibit K562 cell proliferation, we decided to elucidate if DGKalpha could be involved in nuclear signalling and cell cycle regulation. Interestingly, when DGKalpha was silenced in K562 cells, we obtained a cell cycle profile which was a phenocopy of the one obtained by treating the cells with DGK inhibitors I and II, with an important accumulation at G0/G1. Conversely, when we overexpressed DGKalpha, we observed a slight increase in cell proliferation, with a major accumulation in the S phase. Finally, upon overexpression of DGKalpha, the effects of both DGK inhibitor I and DGK inhibitor II were sharply reduced, indicating that both inhibitors were titrated out by DGKa overexpression, and suggesting that this specific isoform was the inhibitor target relevant for the modulation of cell cycle. Altogether, these data demonstrate that DGKalpha modulates K562 cell cycle, putatively by participating to the nuclear PI cycle. The molecular mechanism by which nuclear DGKalpha controls pRb phosphorylation and cell cycle are currently unknown, but they may be of general interest in the hematopoietic system, as similar effects were reported in lymphocytes treated with DGK inhibitors (Flores et al., 1999). The evidences reported in this study may therefore contribute to consider DGKalpha as part of the important machinery regulating $\mathrm{PI}$ metabolism in the nuclear compartment of $\mathrm{K} 562$ cells and, consequently, fundamental processes affecting cell cycle and cell proliferation.

\section{Acknowledgments}

This study was supported by Intesa San Paolo Grant to LC, and by AIRC IG 13524to AG.

Conflict of Interest Statement: All authors declare no competing conflicts of interests 


\section{Bibliography}

Almena M, Andrada E, Liebana R, Merida I. 2013. Diacylglycerol metabolism attenuates T-cell receptor signaling and alters thymocyte differentiation. Cell Death Dis 4:e912.

Bacchiocchi R, Baldanzi G, Carbonari D, Capomagi C, Colombo E, van Blitterswijk WJ, Graziani A, Fazioli F. 2005. Activation of alpha-diacylglycerol kinase is critical for the mitogenic properties of anaplastic lymphoma kinase. Blood 106(6):2175-2182.

Baldanzi G. 2014. Inhibition of diacylglycerol kinases as a physiological way to promote diacylglycerol signaling. Adv Biol Regul 55:39-49.

Baldanzi G, Pighini A, Bettio V, Rainero E, Traini S, Chianale F, Porporato PE, Filigheddu N, Mesturini R, Song S, Schweighoffer T, Patrussi L, Baldari CT, Zhong XP, van Blitterswijk WJ, Sinigaglia F, Nichols KE, Rubio I, Parolini O, Graziani A. 2011. SAP-mediated inhibition of diacylglycerol kinase alpha regulates TCR-induced diacylglycerol signaling. J Immunol 187(11):5941-5951.

Blalock WL, Bavelloni A, Piazzi M, Tagliavini F, Faenza I, Martelli AM, Follo MY, Cocco L. 2011. Multiple forms of PKR present in the nuclei of acute leukemia cells represent an active kinase that is responsive to stress. Leukemia 25(2):236-245.

Blind RD. 2014. Disentangling biological signaling networks by dynamic coupling of signaling lipids to modifying enzymes. Adv Biol Regul 54:25-38.

Bulley SJ, Clarke JH, Droubi A, Giudici ML, Irvine RF. 2015. Exploring phosphatidylinositol 5phosphate 4-kinase function. Adv Biol Regul 57:193-202.

Chiarini F, Grimaldi C, Ricci F, Tazzari PL, Evangelisti C, Ognibene A, Battistelli M, Falcieri E, Melchionda F, Pession A, Pagliaro P, McCubrey JA, Martelli AM. 2010. Activity of the novel dual phosphatidylinositol 3-kinase/mammalian target of rapamycin inhibitor NVP-BEZ235 against T-cell acute lymphoblastic leukemia. Cancer Res 70(20):8097-8107. 
Cocco L, Finelli C, Mongiorgi S, Clissa C, Russo D, Bosi C, Quaranta M, Malagola M, Parisi S, Stanzani M, Ramazzotti G, Mariani GA, Billi AM, Manzoli L, Follo MY. 2015a. An increased expression of PI-PLCbeta1 is associated with myeloid differentiation and a longer response to azacitidine in myelodysplastic syndromes. J Leukoc Biol 98(5):769-780.

Cocco L, Follo MY, Manzoli L, Suh PG. 2015b. Phosphoinositide-specific phospholipase C in health and disease. J Lipid Res 56(10):1853-1860.

D'Santos CS, Clarke JH, Irvine RF, Divecha N. 1999. Nuclei contain two differentially regulated pools of diacylglycerol. Curr Biol 9(8):437-440.

Divecha N, Banfic H, Irvine RF. 1993. Inositides and the nucleus and inositides in the nucleus. Cell 74(3):405-407.

Evangelisti C, Tazzari PL, Riccio M, Fiume R, Hozumi Y, Fala F, Goto K, Manzoli L, Cocco L, Martelli AM. 2007. Nuclear diacylglycerol kinase-zeta is a negative regulator of cell cycle progression in C2C12 mouse myoblasts. FASEB J 21(12):3297-3307.

Flores I, Casaseca T, Martinez AC, Kanoh H, Merida I. 1996. Phosphatidic acid generation through interleukin 2 (IL-2)-induced alpha-diacylglycerol kinase activation is an essential step in IL2-mediated lymphocyte proliferation. J Biol Chem 271(17):10334-10340.

Flores I, Jones DR, Cipres A, Diaz-Flores E, Sanjuan MA, Merida I. 1999. Diacylglycerol kinase inhibition prevents IL-2-induced G1 to S transition through a phosphatidylinositol-3 kinaseindependent mechanism. J Immunol 163(2):708-714.

Follo MY, Manzoli L, Poli A, McCubrey JA, Cocco L. 2015. PLC and PI3K/Akt/mTOR signalling in disease and cancer. Adv Biol Regul 57:10-16.

Giudici ML, Clarke JH, Irvine RF. 2016. Phosphatidylinositol 5-phosphate 4-kinase gamma (PI5P4Kgamma), a lipid signalling enigma. Adv Biol Regul 61:47-50. 
Goto K, Tanaka T, Nakano T, Okada M, Hozumi Y, Topham MK, Martelli AM. 2014. DGKzeta under stress conditions: "to be nuclear or cytoplasmic, that is the question". Adv Biol Regul $54: 242-253$

Irvine RF. 2003. Nuclear lipid signalling. Nat Rev Mol Cell Biol 4(5):349-360.

Lonetti A, Antunes IL, Chiarini F, Orsini E, Buontempo F, Ricci F, Tazzari PL, Pagliaro P, Melchionda F, Pession A, Bertaina A, Locatelli F, McCubrey JA, Barata JT, Martelli AM. 2014. Activity of the pan-class I phosphoinositide 3-kinase inhibitor NVP-BKM120 in T-cell acute lymphoblastic leukemia. Leukemia 28(6):1196-1206.

Los AP, Vinke FP, de Widt J, Topham MK, van Blitterswijk WJ, Divecha N. 2006. The retinoblastoma family proteins bind to and activate diacylglycerol kinase zeta. J Biol Chem 281(2):858-866.

Matsubara T, Ikeda M, Kiso Y, Sakuma M, Yoshino K, Sakane F, Merida I, Saito N, Shirai Y. 2012. cAbl tyrosine kinase regulates serum-induced nuclear export of diacylglycerol kinase alpha by phosphorylation at Tyr-218. J Biol Chem 287(8):5507-5517.

Matsubara T, Shirai Y, Miyasaka K, Murakami T, Yamaguchi Y, Ueyama T, Kai M, Sakane F, Kanoh H, Hashimoto T, Kamada S, Kikkawa U, Saito N. 2006. Nuclear transportation of diacylglycerol kinase gamma and its possible function in the nucleus. J Biol Chem 281(10):6152-6164.

Merida I, Avila-Flores A, Merino E. 2008. Diacylglycerol kinases: at the hub of cell signalling. Biochem J 409(1):1-18.

Payrastre B, Nievers M, Boonstra J, Breton M, Verkleij AJ, Van Bergen en Henegouwen PM. 1992. A differential location of phosphoinositide kinases, diacylglycerol kinase, and phospholipase C in the nuclear matrix. J Biol Chem 267(8):5078-5084.

Poli A, Faenza I, Chiarini F, Matteucci A, McCubrey JA, Cocco L. 2013. K562 cell proliferation is modulated by PLCbeta1 through a PKCalpha-mediated pathway. Cell Cycle 12(11):17131721. 
Poli A, Mongiorgi S, Cocco L, Follo MY. 2014a. Protein kinase C involvement in cell cycle modulation. Biochem Soc Trans 42(5):1471-1476.

Poli A, Ramazzotti G, Matteucci A, Manzoli L, Lonetti A, Suh PG, McCubrey JA, Cocco L. 2014b. A novel DAG-dependent mechanism links PKCa and Cyclin B1 regulating cell cycle progression. Oncotarget 5(22):11526-11540.

Raben DM, Tu-Sekine B. 2008. Nuclear diacylglycerol kinases: regulation and roles. Front Biosci 13:590-597.

Ruffo E, Malacarne V, Larsen SE, Das R, Patrussi L, Wulfing C, Biskup C, Kapnick SM, Verbist K, Tedrick P, Schwartzberg PL, Baldari CT, Rubio I, Nichols KE, Snow AL, Baldanzi G, Graziani A. 2016. Inhibition of diacylglycerol kinase alpha restores restimulation-induced cell death and reduces immunopathology in XLP-1. Sci Transl Med 8(321):321ra327.

Sato M, Liu K, Sasaki S, Kunii N, Sakai H, Mizuno H, Saga H, Sakane F. 2013. Evaluations of the selectivities of the diacylglycerol kinase inhibitors R59022 and R59949 among diacylglycerol kinase isozymes using a new non-radioactive assay method. Pharmacology 92(1-2):99-107. Shulga YV, Topham MK, Epand RM. 2011. Regulation and functions of diacylglycerol kinases. Chem Rev 111(10):6186-6208.

Tanaka T, Tsuchiya R, Hozumi Y, Nakano T, Okada M, Goto K. 2016. Reciprocal regulation of p53 and NF-kappaB by diacylglycerol kinase zeta. Adv Biol Regul 60:15-21.

Topham MK, Bunting M, Zimmerman GA, Mclntyre TM, Blackshear PJ, Prescott SM. 1998. Protein kinase $\mathrm{C}$ regulates the nuclear localization of diacylglycerol kinase-zeta. Nature 394(6694):697-700.

Tu-Sekine B, Goldschmidt H, Raben DM. 2015. Diacylglycerol, phosphatidic acid, and their metabolic enzymes in synaptic vesicle recycling. Adv Biol Regul 57:147-152.

Vann LR, Wooding FB, Irvine RF, Divecha N. 1997. Metabolism and possible compartmentalization of inositol lipids in isolated rat-liver nuclei. Biochem J 327 ( Pt 2):569-576. 
Wada I, Kai M, Imai S, Sakane F, Kanoh H. 1996. Translocation of diacylglycerol kinase alpha to the nuclear matrix of rat thymocytes and peripheral T-lymphocytes. FEBS Lett 393(1):48-52. 


\section{Figures}

Figure 1. DGKalpha is highly located located into the nuclear compartment of K562 cells. a) Cell cycle analyses showing $\mathrm{K} 562$ cell synchronization at the G1/S, G2/M and G0/G1 phase of the cell cycle. b) Nuclei and cytoplasms of synchronized K562 cells were separated. Immunoblot assay indicated DGKalpha as highly present in the nuclear compartment of K562 cells, slightly peaking at the $\mathrm{G} 2 / \mathrm{M}$ phase.

Figure 2. DGK inhibition drives cells to accumulate into G0/G1 and to changes in Cyclin and ppRb levels. a) K562 cell proliferation was quantified after $48 \mathrm{~h}$ treatment with DGK inhibitor I (DI) and DGK inhibitor II (DII) at different final concentrations (DI 5-10-20-30-50 $\mu \mathrm{M}$ / DII 5-10-20-30-50 $\mu \mathrm{M})$. Cell growth was significantly down-regulated starting from $20 \mu \mathrm{M}$ to $50 \mu \mathrm{M}$ if compared to control cells (Ctrl). Data were collected by three independent analyses \pm SD. ${ }^{* * *} p<0.05$ vs. Ctrl. b) Cell counting experiments confirmed that cell proliferation was slowed down by DGK inhibition (DI 5-10-20-30 $\mu \mathrm{M}$ / DII 5-10-20-30 $\mu \mathrm{M}$ / Control). Data were collected by three independent analyses $\pm \mathrm{SD}$. $* * * p<0.05$ vs. Ctrl. c) Cell cycle analysis showed accumulation at the G0/G1 phase after treatment with DGK inhibitors I and II at $20 \mu \mathrm{M}$ for 48h (DGK inh I $20 \mu \mathrm{M} 48 \mathrm{~h}$ / DGK inh II $20 \mu \mathrm{M}$ 48h). Data were collected by three independent analyses \pm SD. ${ }^{* *} p<0.05$ vs. Ctrl. DGK inhibition led to minor G1/S transition if compared with control cells (Ctrl 48h). d) Western blot analyses of proteins involved in cell cycle regulation indicated that DGK inhibition (DGK inhibitor I 48h / DGK inhibitor II 48h) induced a slight increase of Cyclin D3, a minor expression of Cyclin B1 and a diminished phosphorylation of $\mathrm{pRb}$.

Figure 3. DGK signaling inhibition did not lead to changes in the expression of autophagy markers. K562 cells were treated with DGK inhibitor I and DGK inhibitor II for 48 hours (DGK inhibitor I 48h - DGK inhibitor II 48h) and the expression of Beclin 1 and LC-3b, markers of autophagy, was investigated. No changes in their levels were detected as compared with control cells (Control).

Figure 4. Cell cycle arrest at G0/G1 phase is not followed by apoptosis. a) Cells were treated for 48 hours with DGK inhibitor I and II (DGK inhibitor I 48h / DGK inhibitor II 48h and Annexin-V assay 
was performed. No changes in the percentage of apoptotic cells were detected comparing DGK inhibitors treatments with normal proliferating control cells (Control 48h). b) Protein expression of caspase 3 and caspase 9 (both total and cleaved forms) showed no differences among the different samples (DGK inhibitor I 48h/ DGK inhibitor II 48h/ Control 48h).

Figure 5. Nuclear DGKalpha modulation is involved in $\mathrm{K} 562$ proliferation and cell cycle regulation. a) Knock-down of DGKalpha was obtained using specific siRNA (DGKalpha KD), whereas control cells were transfected with a mix of scrambled siRNAs (Scrambled). Immunoblot and RealTime PCR analyses confirmed both the gene and protein silencing. b) Cells were transfected with DGKalpha siRNA (DGKalpha KD) and with Scrambled siRNAs (Scrambled), seeded at a final cell density of $2.5 \times 10^{4}$ cells $/ \mathrm{ml}$ and counted after 24-48-72 hours. Slow-down of cell proliferation was detected only in DGKalpha KD cells. c) 48 hours after transfection, cells with DGKalpha knockdown (DGKalpha KD 48h) showed an accumulation at the G0/G1 phase, if compared with the control (Scrambled 48h). d) Overexpression of DGKalpha was obtained using a specific vector encoding a wild type protein (DGKalpha OV). As control, cells were transfected with an empty vector (Empty). Immunoblot and Real-Time PCR analyses confirmed the overexpression. e) Cells were transfected with DGKalpha (DGKalpha OV) and with an empty vector (Empty Vector), then seeded at a final cell density of $2.5 \times 10^{4}$ cells $/ \mathrm{ml}$ and counted after 24-48-72 hours. A slight increase of cell proliferation was detected in DGKalpha OV cells. f) 48 hours after transfection, cell cycle profile of cells overexpressing DGKalpha (DGKalpha OV) showed a higher percentage of cells progressing into $\mathrm{S}$ phase, if compared with the control (Empty). Data were collected by three independent analyses \pm SD. ${ }^{*} p<0.05$.

Figure 6. Overexpression of DGKalpha partially restores the normal cell cycle phenotype of cells treated with DGK inhibitor I and DGK inhibitor II. K562 cells were transfected with a DGKalpha vector and with an empty vector as control. 24 hours after the procedure, cells were treated with both DGK inhibitor I and II for other 24 hours and cell cycle profile was analyzed. Cells with DGKalpha overexpression (DGKalpha OV + DGK inh I / DGKalpha OV + DGK inh II) showed a diminished G0/G1 accumulation after DGK inhibition as compared with cells transfected with the empty vector (Empty + DGK inh I / Empty + DGK inh II), indicating that DGKalpha was able to 
attenuate the effects of DGK inhibitors I and II. Data were collected by three independent analyses and cell cycle experiments were analyzed by Student's t-test. * $p<0.05$. 

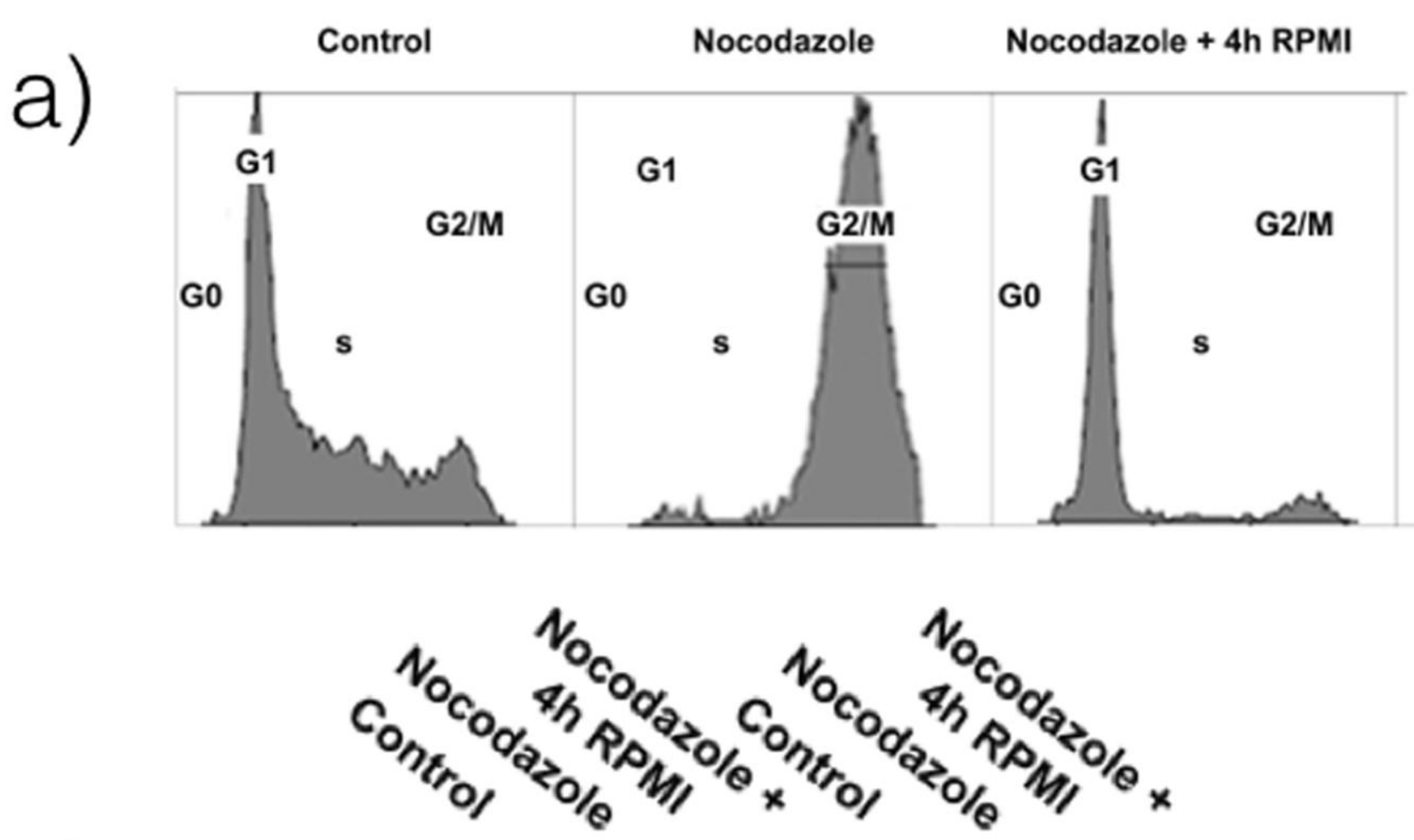

b)

$$
\text { NUCLEI CYTOPLASMS }
$$

80 kDa

55 kDa

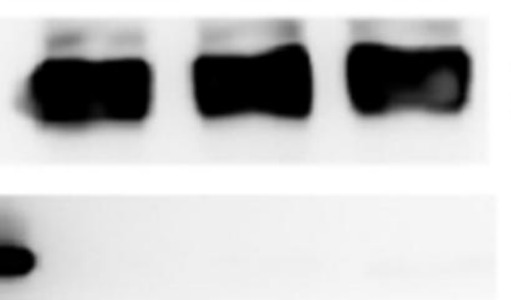

$\beta$-tubulin

17 kDa

H2A

Figure 1 
a)

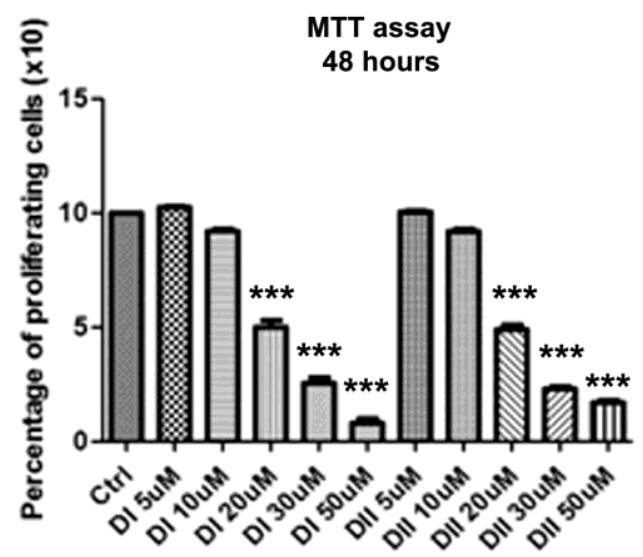

b)

$$
\text { Cell proliferation assay }
$$
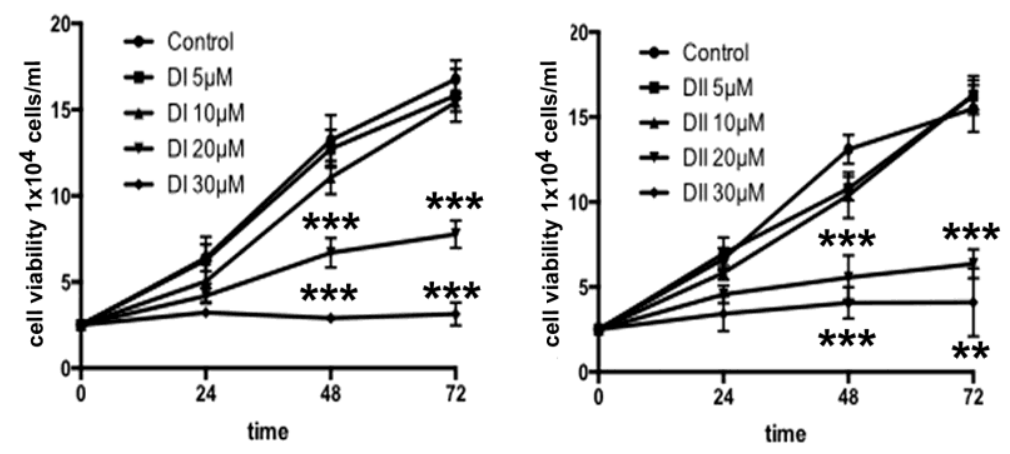

c)

Cell cycle analyses

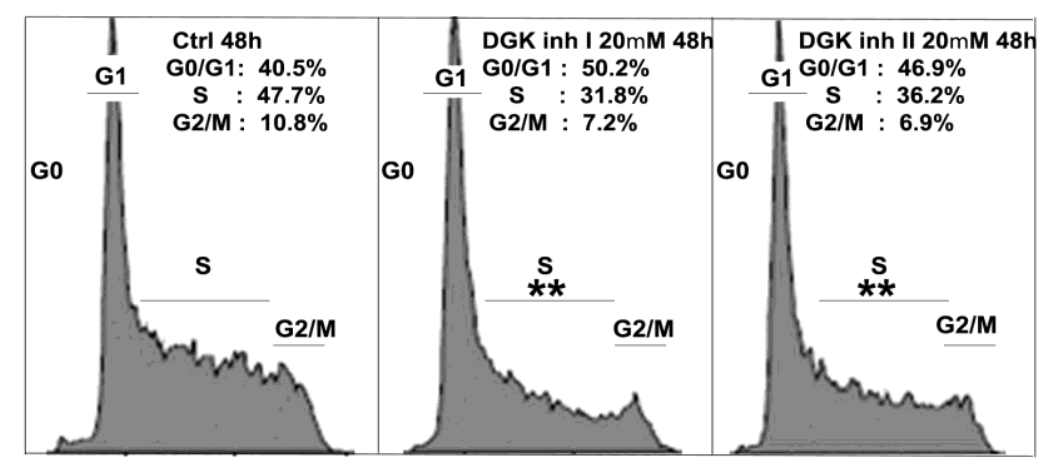

d)

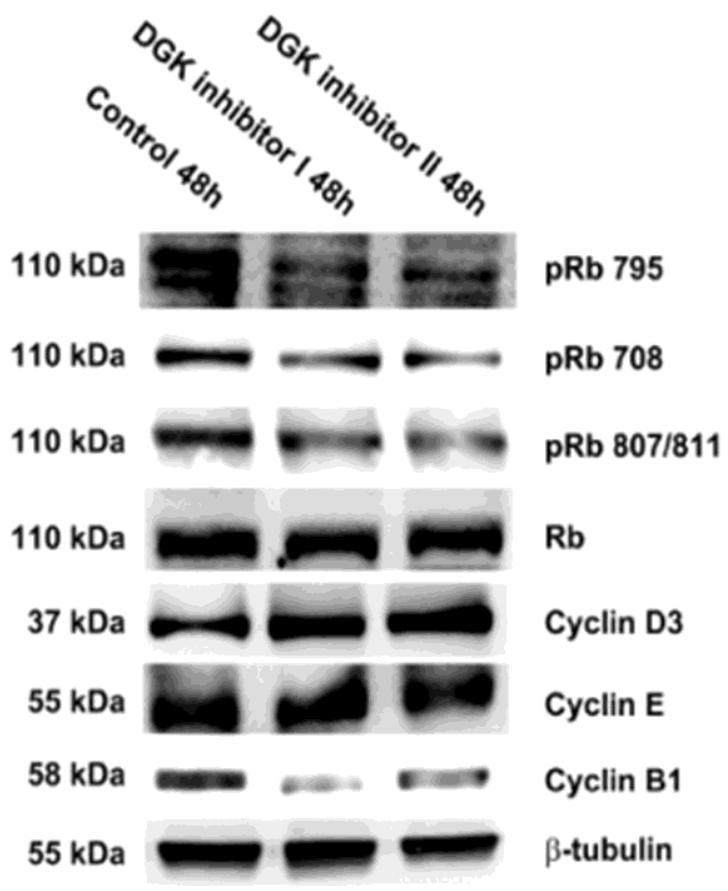

Figure 2 


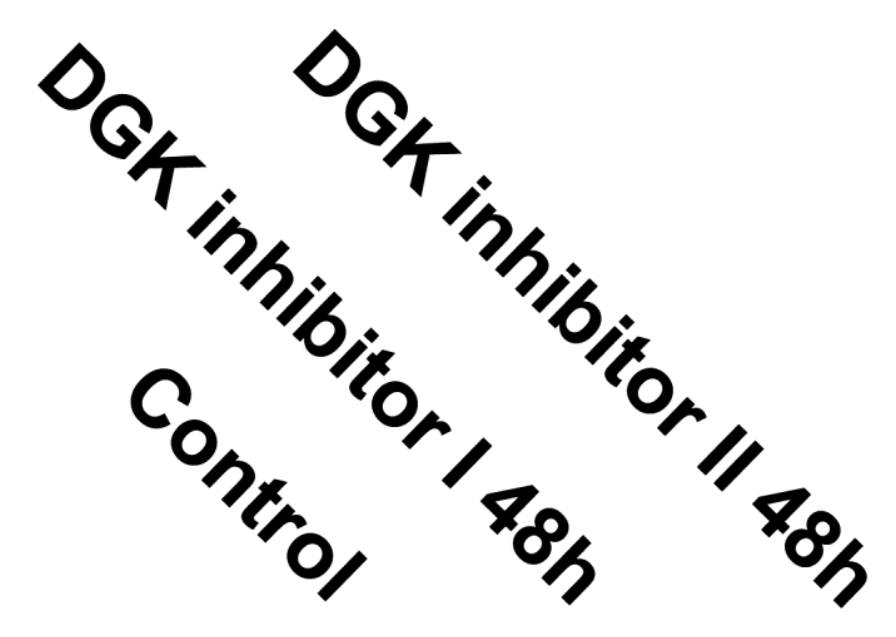

$52 \mathrm{kDa}$

Beclin 1

$17 \mathrm{kDa}$

LC3-b

$55 \mathrm{kDa}$

$\beta$-tubulin

Figure 3 
a) Annexin assay
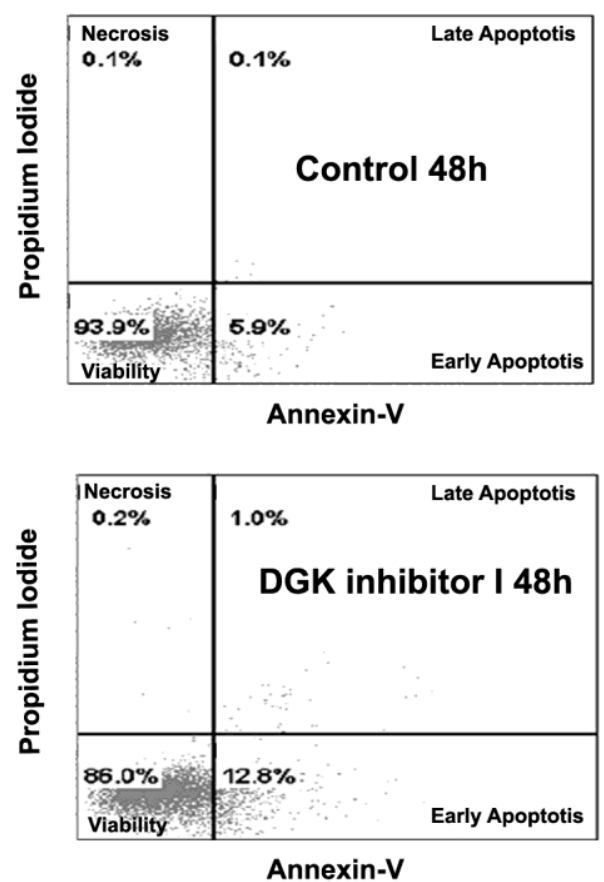

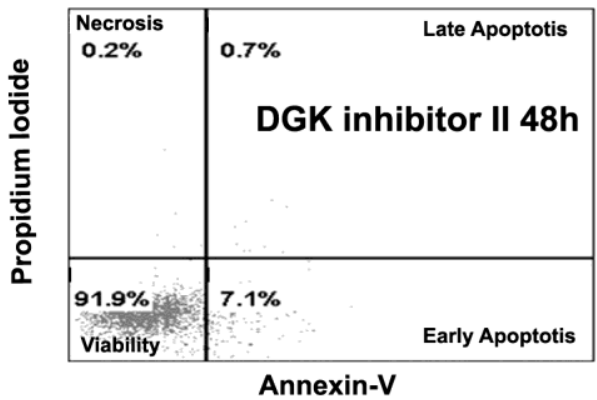

b)

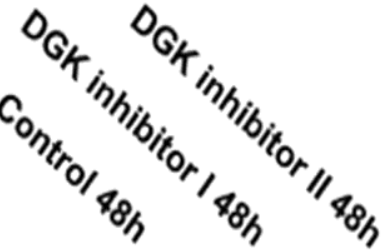

$35 \mathrm{kDa}$

Caspase 3

$19 \mathrm{kDa}$

$50 \mathrm{kDa}$

Caspase 9

$39 \mathrm{kDa}$

$55 \mathrm{kDa}$ $\beta$-tubulin

\section{Figure 4}


a)

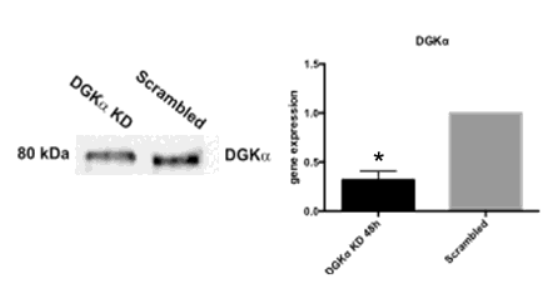

d)

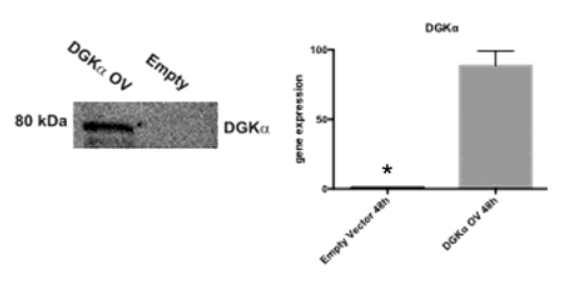

b)

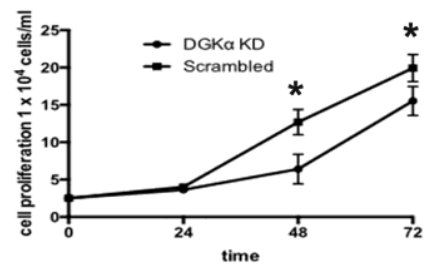

c)

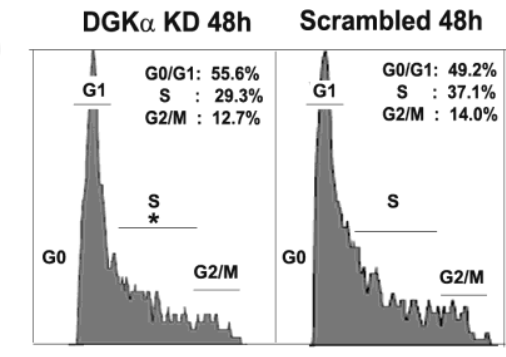

e)

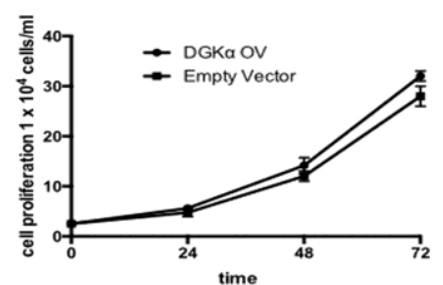

f)

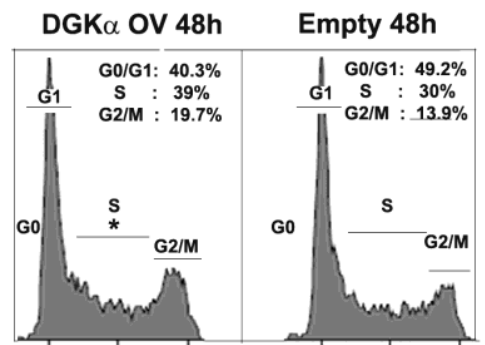

Figure 5 


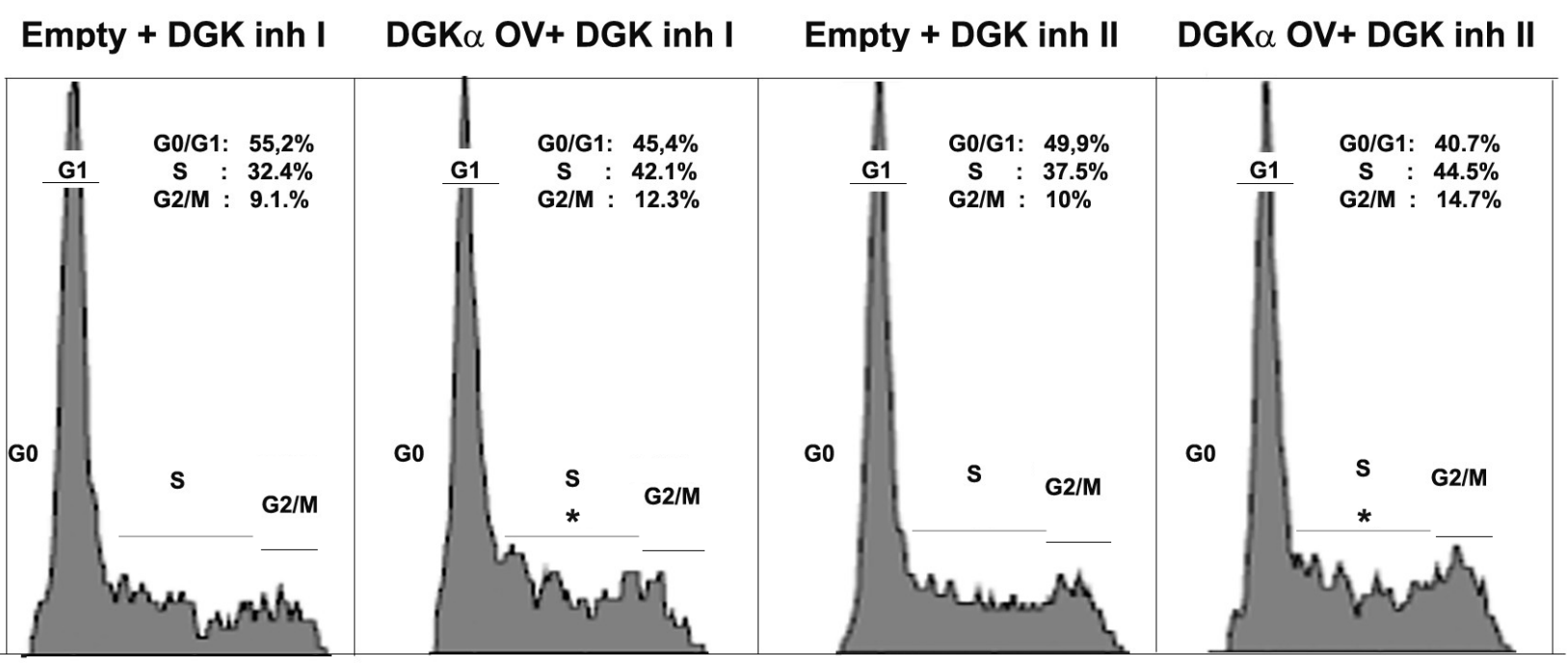

Figure 6 